\title{
Understanding the benign nature of natural SIV infection: Implications for AIDS pathogenesis Guido Silvestri*
}

Address: Department of Pathology, University of Pennsylvania School of Medicine, Philadelphia, Pennsylvania, USA

* Corresponding author

from 2006 International Meeting of The Institute of Human Virology

Baltimore, USA. 17-2I November, 2006

Published: 21 December 2006

Retrovirology 2006, 3(SuppI I):S94 doi:I0.1 I86/I742-4690-3-SI-S94

(C) 2006 Silvestri; licensee BioMed Central Ltd.

Simian immunodeficiency viruses (SIV) infections of natural hosts, including sooty mangabey (SM), African green monkeys (AGMs), and many other African non-human primate species, typically do not induce CD4+ T cell depletion and acquired immunodeficiency syndrome (AIDS) despite chronic high levels of virus replication. In contrast, SIV infection of non-natural hosts, such as several macaque species, induces a disease that resembles AIDS in humans. The mechanisms underlying the lack of disease progression in SIV-infected SMs are incompletely understood, but certainly reflect a complex evolutionary adaptation whereby the host immune system is not significantly damaged by the highly replicating virus. It is now widely recognized that a better understanding of these mechanisms may provide clues to the pathogenesis of immunodeficiency in HIV-infected humans. In this presentation we will review and discuss: (1) the evidence supporting a key role for the absence of generalized immune activation in maintaining natural SIV hosts disease-free; (2) the potential mechanisms underlying the different level of immune activation in natural versus non-natural HIV/SIV hosts; and (3) the similarities and differences in the way SIV infection affects the mucosal immune system in natural versus non-natural hosts. 